\title{
ARTIGO
}

\section{Condutas na Estenose da Carótida}

Marcia Maiumi Fukujima*

Alberto Alain Gabbai **

\section{RESUMO}

Estenose de carótida pode ser assintomática ou sintomática, manifestando-se por ataque isquêmico transitório ou infarto cerebral. Quando assintomática evolui com risco de $10 \%$ ao ano de evento clínico vascular cerebral. O principal exame para diagnóstico é o ultra-som doppler de carótidas, porém não se recomenda esse exame rotineiramente para a população geral, reservando-se para os casos de alto risco vascular. Nas últimas duas décadas têm sido realizados vários estudos randomizados para definição de condutas nas estenoses carotídeas Com base nesses estudos, recomenda-se endarterectomia para estenose sintomática $\geq 70 \%$ se não houver contra-indicações ao procedimento, e também para estenoses assintomáticas $\geq 60 \%$, porém considerando-se cautelosamente o risco cirúrgico do serviço. Caso não haja indicação de cirurgia, os pacientes devem submeter-se a tratamento clínico, que no caso de estenose assintomática constitui-se do controle dos fatores de risco aterotrombótico e nos casos de estenose sintomática, além do controle dos fatores de risco, utiliza-se droga antiagregante plaquetária.

\section{UNITERMOS}

Estenose da carótida, doença vascular cerebral, endarterectomia.
Neurologista do Setor de Urgências e do Setor de Doenças Neurovasculares da Disciplina de Neurologia da Escola Paulista de Medicina - UNIFESP.

** Professor Titular da Disciplina de Neurologia da Escola Paulista de Medicina - UNIFESP.

\section{INTRODUÇÃO}

A estenose da carótida é responsável por cerca de $20 \%$ dos acidentes vasculares cerebrais oclusivos do território carotídeo' ${ }^{1}$. O principal processo patológico é a aterosclerose do sistema arterial cerebral. Trata-se de um processo degenerativo localizado na camada íntima dos vasos, caracterizado por acúmulo de lípides plasmáticos, fibras do tecido conjuntivo e células locais e circulantes. A formação da placa aterosclerótica leva ao estreitamento da luz vascular e à redução do fluxo, o que caracteriza a estenose da carótida, podendo complicar com ulceração da placa levando à trombose e embolização distal ${ }^{2.3}$.

Endarterectomia da carótida tem sido realizada desde a década de 50, porém sua indicação tem sofrido modificações conforme surgem trabalhos controlados. Na década de 70, nos Estados Unidos, faziam-se cerca de 15.000 cirurgias anuais, esse número aumentou para 107.000 em 1985. Na década de 80 começaram os primeiros estudos controlados sobre endarterectomia de carótida e no final dessa década surgiam os primeiros resultados dos estudos, fazendo o número de cirurgias cair para 45.000 em 1989 , principalmente devido à definição das indicações precisas e da consideração do risco paciente-cirúrgico, passando a ser realizada somente no grupo de pacientes que realmente se beneficiariam com o procedimento sem correr riscos adicionais; em 1997, foram realizadas 110.000 a 115.000 cirurgias tecnicamente aperfeiçoadas e mais seguras em relação às de 20 anos atrás ${ }^{4}$.

A estenose carotídea pode ser assintomática ou sintomática.

A estenose assintomática da carótida (EAC) é aquela em que não há sintoma neurológico relacionado. Nos Estados Unidos devem existir aproximadamente 2 milhões de indivíduos com EAC. A incidência de EAC na faixa etária de 50 a 59 anos é de $1,7 \%$ para homens e zero para mulheres; na faixa etária de 60 a 69 anos é de $4,2 \%$ para homens e de 1,8\% para mulheres $^{5}$. Não temos estatísticas nacionais disponíveis.

Para EAC $>75 \%$, estima-se incidência de AVC de $3 \%$ ao ano, e se forem somados casos de ataque isquêmico transitório, a incidência chega a $10 \%$ ao ano; além do risco vascular cerebral, os portadores de EAC têm risco elevado para eventos coronarianos (cerca de $8,5 \%$ ao ano) e morte de origem vascular

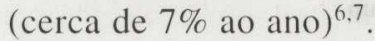


Um elemento propedêutico para o diagnóstico de EAC é o sopro carotídeo, que é preditivo de acidente vascular cerebral isquêmico (AVCI) em $76 \%$ dos casos.

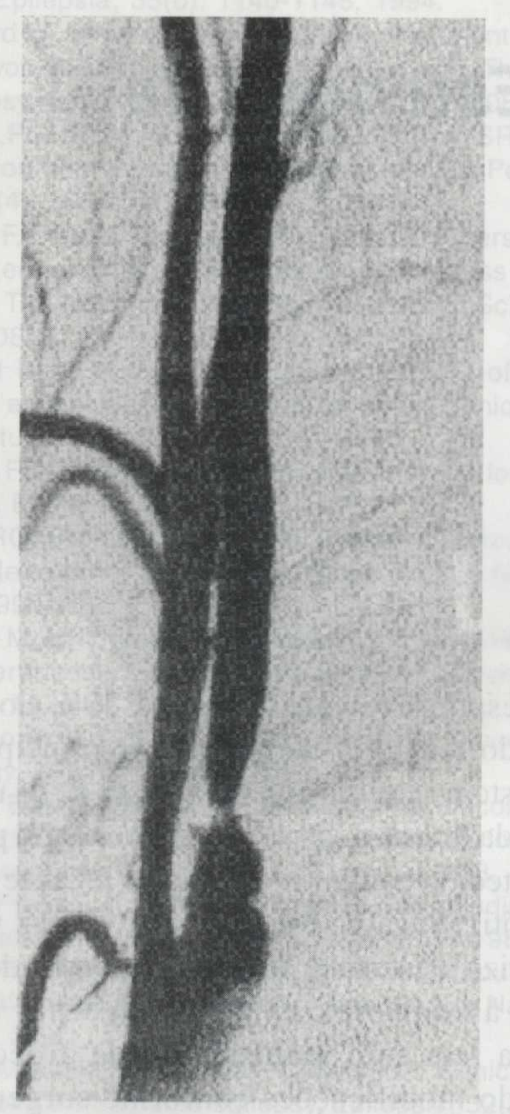

FIGURA 1

Bifurcação da artéria caródida comum - aspecto ultra-sonográfico.
Porém, o sopro carotídeo tem baixa especificidade para predizer a EAC, estando presente em $4 \%$ da população geral sem doença vascular e em até $10 \%$ em indivíduos com mais que 60 anos, e destes menos de $1 \%$ tem estenoses $>75 \% \%^{8,9}$. Nas estenoses graves, há importante redução do fluxo carotídeo, fazendo com que o sopro torne-se inaudível. O diagnóstico é realizado com auxílio de métodos de imagem, principalmente exame ultrasonográfico (Figura 1) com fluxometria das carótidas, angiografia dos vasos cerebrais (Figura 2), angiorressonância magnética dos vasos cerebrais e reconstrução tomográfica de vasos cerebrais.

Apesar da grande importância da EAC, não se justifica a recomendação de exame de imagem das

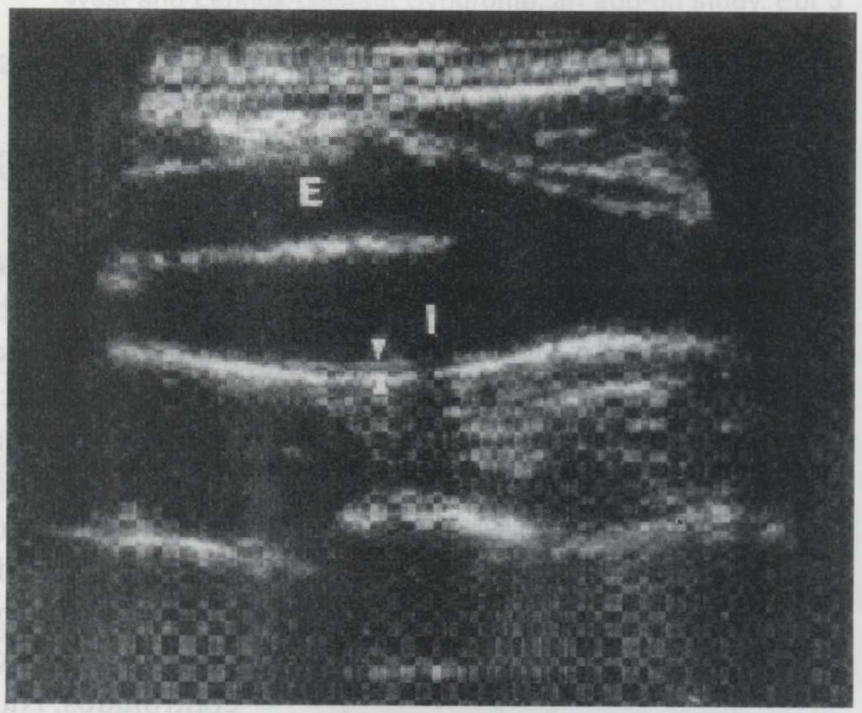

FIGURA 2

Estenose da artéria carótida interna - angiografia.

\section{TABELA 1}

Resultados dos principais estudos controlados de estenose assintomática da carótida, considerando a mortalidade perioperatória e a redução de risco para diferentes intervalos de tempo

\begin{tabular}{lccccc}
\hline & \multicolumn{2}{c}{ número de pacientes } & \multicolumn{2}{c}{ mortalidade (\%) } & \multicolumn{2}{c}{ redução do risco } \\
\cline { 2 - 6 } Estudo - período & cirúrgico & clínico & cirúrgico & clínico & significante \\
\hline ACAS (1987-93) & 825 & 834 & 0,4 & 0,1 & $5,9 \%$ em 5 anos \\
VACS (1983-91) & 211 & 233 & 1,9 & 0,4 & nenhuma (4 anos) \\
CASANOVA (1982-88) & 206 & 204 & 1,5 & 0,5 & nenhuma (3 anos) \\
MACES (1987-90) & 36 & 35 & 0,0 & 0,0 & nenhuma (2 anos) \\
\hline
\end{tabular}

ACAS = Asymptomatic Carotid Atherosclerosis Study;

VACS = Veterans Affair Cooperative Study Group;

CASANOVA $=$ CASANOVA Study Group;

MACE = Mayo Asymptomatic Carotid Endarterectomy Study Group. 
TABELA 2

Resultados dos principais estudos controlados de estenose sintomática da carótida, considerando a mortalidade perioperatória e a redução de risco para diferentes intervalos de tempo

\begin{tabular}{|c|c|c|c|c|c|}
\hline \multirow[b]{2}{*}{ Estudo - período } & \multicolumn{2}{|c|}{ número de pacientes } & \multicolumn{2}{|c|}{ mortalidade (\%) } & \multirow{2}{*}{$\begin{array}{c}\text { redução do risco } \\
\text { significante }\end{array}$} \\
\hline & cirúrgico & clínico & cirurgico & clínico & \\
\hline NASCET (1988-91) & 328 & 331 & 0,6 & 0,3 & $17 \%$ em 2 anos \\
\hline VACS (1988-91) & 92 & 101 & 3,3 & 0,0 & $11,7 \%$ em 1 ano \\
\hline ECST 0-29\% (1981-91) & 219 & 155 & 1,4 & 0,0 & nenhuma em 3 anos \\
\hline ECST $30-49 \%(1981-95)$ & 389 & 259 & 0.8 & 0,0 & nenhuma em 4 anos \\
\hline ECST $50-69 \%(1981-95)$ & 570 & 372 & 1,4 & 0,0 & nenhuma em 4 anos \\
\hline ECST 70-99\% (1981-91) & 455 & 323 & 0,9 & 0,0 & $5 \%$ em 3 anos \\
\hline
\end{tabular}

NASCET = North American Symptomatic Group Endarterectomy Trial;

VACS $=$ Veterans Affair Cooperative Study Group;

ECST $=$ European Carotid Artery Surgery Trial.

\section{QUADRO 1}

Recomendações de endarterectomia nas EAC

\section{Estenose assintomática da carótida - endarterectomia}

A) Comprovadamente benéfica:

- estenose assintomática $<60 \%$ confirmada por arteriografia (se morbidade e mortalidade perioperatória $<3 \%$ e pacientes selecionados criteriosamente com controle agressivo dos fatores de risco)

B) Benefício incerto:

- estenose $>75 \%$ com alto risco paciente-cirúrgico (índice de mortalidade e morbidade $>3 \%$ )

- cirurgia combinada de carótida e coronária

- lesão ulcerada sem estenose hemodinâmica significante.

\section{QUADRO 2}

Recomendações para endarterectomia da carótida nas estenoses sintomáticas

Estenose sintomática da carótida - endarterectomia

A) Comprovadamente benéfica:

- AIT hemisférico ou retiniano

- AVC sem incapacidade

- estenose grave (70\%-99\%) da carótida interna ipsilateral

B) Aceitável, mas não comprovada (representam boas indicações para endarterectomia, baseadas em dados ainda não comprovados cientificamente)

- AIT (isolada, múltipla ou em crescendo) nos últimos 6 meses com estenose $>70 \%$

- AVC progressivo com estenose $>70 \%$

- AVC moderado ou leve e estenose $50 \%-69 \%$

- endarterectomia ipsilateral combinada com revascularização coronariana em paciente com TIA e estenose unilateral ou bilateral $\geq 70 \%$ 


\section{QUADRO 2}

(continuação)

C) Benefício incerto (baseado em dados ainda insuficientes para cálculo do índice de risco e benefício)

- AIT ou AVC leve com estenose $<50 \%$

- AIT com estenose $<70 \%$ combinada com revascularização coronariana

- AVC moderado ou progressivo com estenose $<69 \%$

- sintomas de isquemia globais

- estenose ipsilateral $>75 \%$, mas contralateral $<75 \%$

- dissecção da carótida interna com sintomas persistentes em vigência de heparina

- oclusão aguda da carótida dentro de 6 horas, evoluindo para AIT ou AVC leve

D) Comprovadamente imprópria (os dados mostram que os riscos cirúrgicos se sobrepõem aos benefícios)

- AIT único, AVC moderado ou progressivo com estenose $<50 \%$, sem uso de aspirina

- sintomas de isquemia global com estenose $<50 \%$

- dissecção da carótida interna assintomática em vigência de heparina; morbidade e mortalidade cirúrgica $>5 \%$

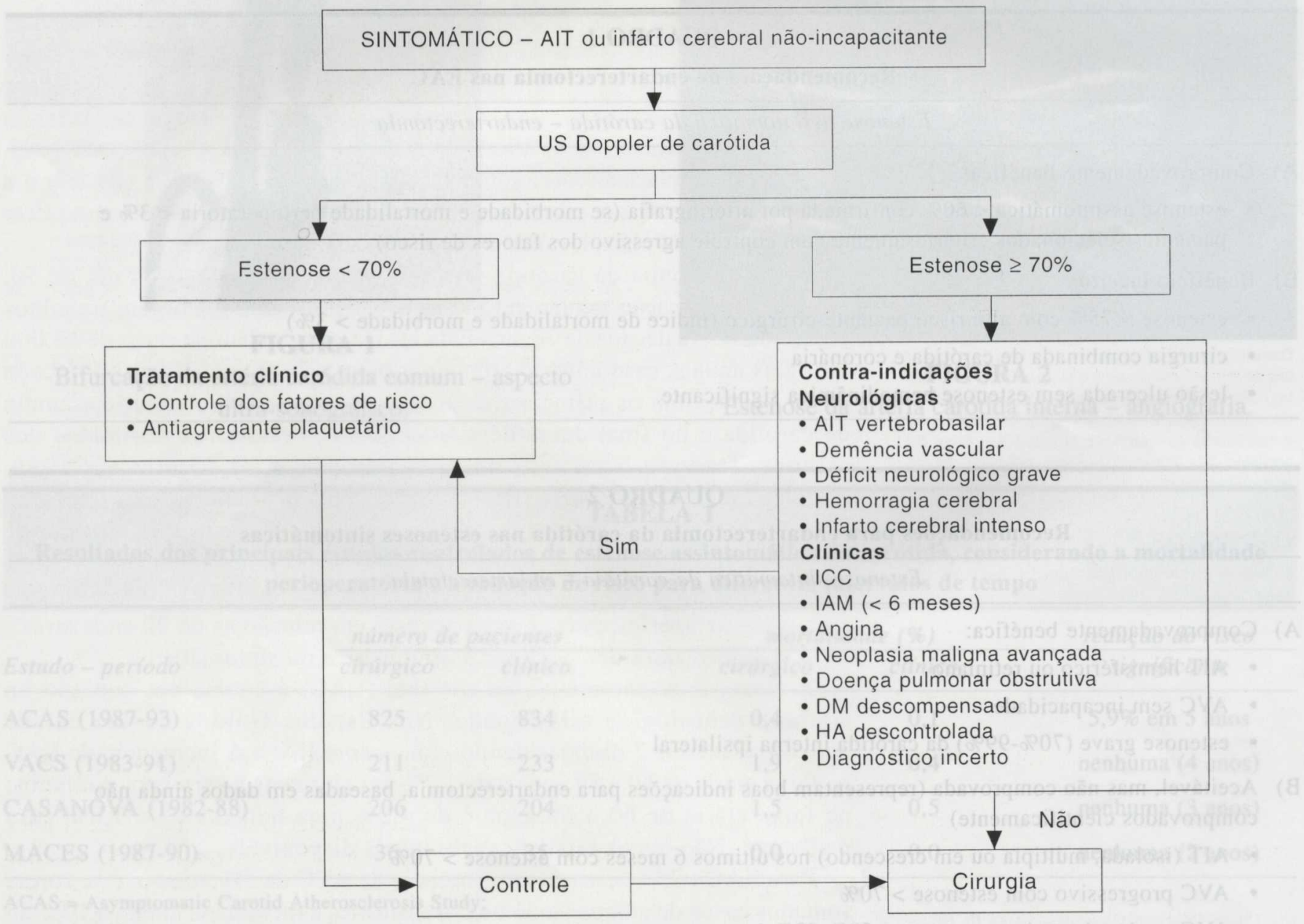

FIGURA 3

Algoritmo para estenose sintomática da carótida. 


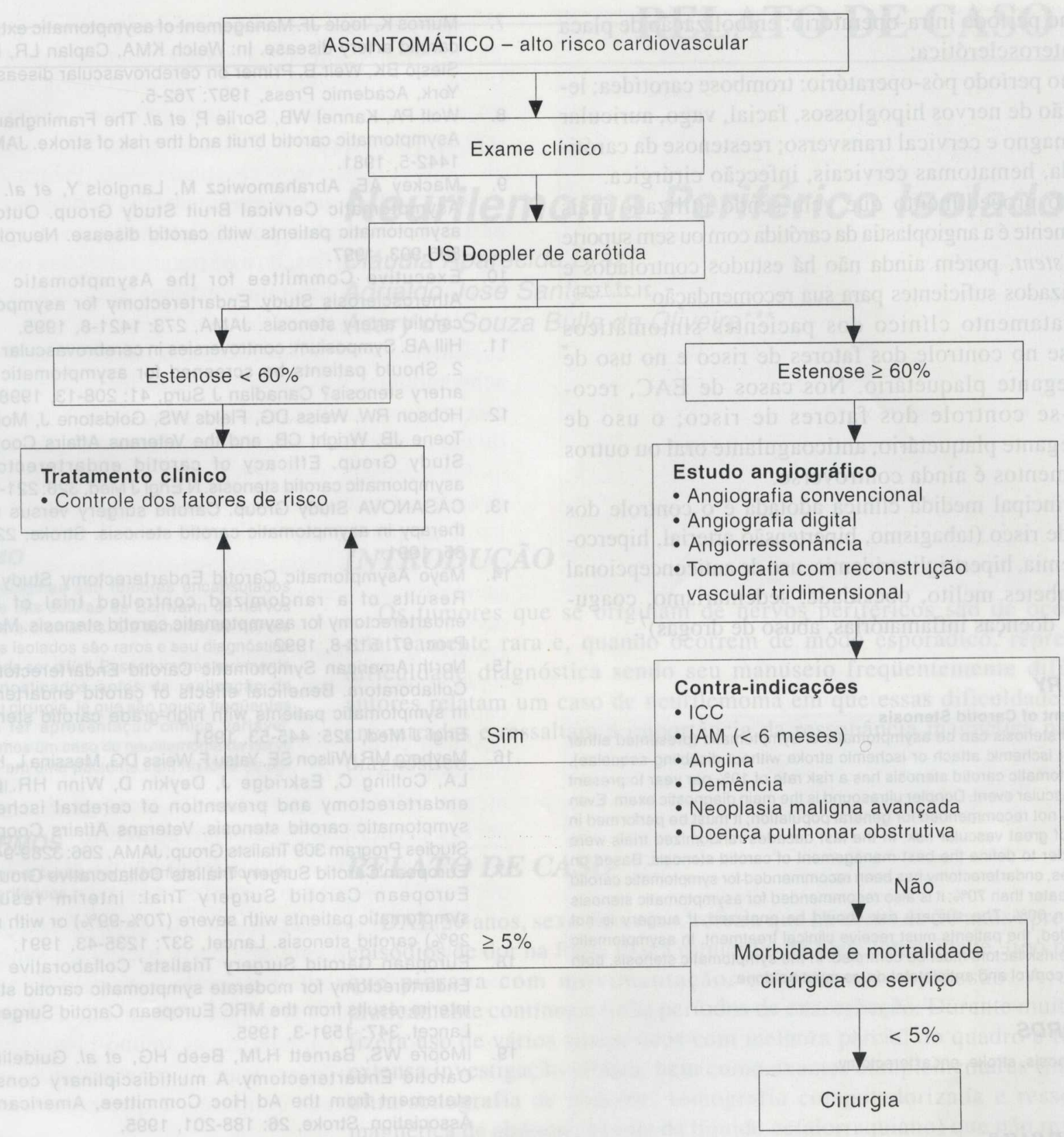

FIGURA 4

Algoritmo para estenose assintomática da carótida.

carótidas de maneira rotineira ao nível populacional, sendo este realizado criteriosamente em casos individuais de pacientes de alto risco cardiovascular, isto é, naqueles portadores de um conjunto de fatores de risco, tais como hipertensão arterial, diabetes melito, dislipidemia, tabagismo, obesidade, entre outros. O rastreamento populacional necessitaria de realização de exames em 850 a 1.700 pessoas (podendo chegar a 8.500 pessoas) para prevenir um episódio de $\mathrm{AVC}^{10,11}$.
Os principais estudos controlados e respectivos resultados são apresentados nas tabelas 1 e $2^{10.11 .12,13,14,15,16,17,18}$.

Com base nesses estudos, algumas recomendações foram adotadas a partir de consensos da American Heart Association $(1995)^{19}$ e foram modificadas pela Canadian Society for Vascular Surgery $(1998)^{20}$, quadros 1 e 2 e figuras 3 e 4.

A endarterectomia é a cirurgia de escolha para os casos selecionados. As complicações mais comuns deste procedimento são: 
- no período intra-operatório: embolização de placa aterosclerótica;

- no período pós-operatório: trombose carotídea; lesão de nervos hipoglossos, facial, vago, auricular magno e cervical transverso; reestenose da carótida, hematomas cervicais, infecção cirúrgica.

Outro procedimento que vem sendo utilizado mais recentemente é a angioplastia da carótida com ou sem suporte interno stent, porém ainda não há estudos controlados e randomizados suficientes para sua recomendação $0^{21,22,23,24}$.

O tratamento clínico dos pacientes sintomáticos baseia-se no controle dos fatores de risco e no uso de antiagregante plaquetário. Nos casos de EAC, recomenda-se controle dos fatores de risco; o uso de antiagregante plaquetário, anticoagulante oral ou outros medicamentos é ainda controverso.

A principal medida clínica adotada é o controle dos fatores de risco (tabagismo, hipertensão arterial, hipercolesterolemia, hipertrigliceridemia, uso de anticoncepcional oral, diabetes melito, obesidade, sedentarismo, coagulopatias, doenças inflamatórias, abuso de drogas $)^{7}$.

\section{SUMMARY}

\section{Management of Carotid Stenosis}

The carotid stenosis can be asymptomatic or symptomatic (presented either by transient ischemic attach or ischemic stroke without disabling sequelae). The asymptomatic carotid stenosis has a risk rate of $10 \%$ per year to present a clinical vascular event. Doppler ultrasound is the main diagnostic exam. Even though, it is not recommended for general population; it must be performed in the cases of great vascular risk. In the last decades randomized trials were made in order to define the best management of carotid stenosis. Based on these studies, endarterectomy has been recommended for symptomatic carotid stenosis greater than $70 \%$; it is also recommended for asymptomatic stenosis greater than $60 \%$. The surgery risk should be analyzed. If surgery is not recommended, the patients must receive clinical treatment. In asymptomatic stenosis the risk factors must be controled. In the symptomatic stenosis, both risk factors control and antiplatelet drugs must be done.

\section{KEY WORDS}

Carotid stenosis, stroke, enciarterectomy.

\section{Referências}

1. DeGraba TJ, Fisher M, Yatsu FM. Atherogenesis and strokes. In: Barnett HJM, Mohr JP, Stein BM, Yatsu F. StrokePathophysiology, Diagnosis and management. 2nd ed. New York, Churchill Livingstone, 1992: 29-48.

2. Ross $R$. The pathogenesis of atherosclerosis - an update . $N$ Engl Med J, 314: 488-500, 1986.

3. Garcia J, Ho KL. Carotid Atherosclerosis - definition, pathogenesis, and clinical Significance. Neuroimaging Clin N Am, 6: 801-10, 1996.

4. Robertson J. Carotid endarterectomy. In: Welch KMA, Caplan LR, Reis DJ, Siesjö BK, Weir B. Primer on cerebrovascular diseases. New York, Academic Press, 1997: 582-6.

5. WilleitJ, Kiechl S. Prevalence and risk factors of asymptomatic extracranial carotid artery atherosclerosis. A population-based study. Arterioscler Thromb, 13: 661-8, 1993.

6. Norris JW, Zhu CZ, Borstein NM, Chambers BR. Vascular risks of asymptomatic carotid stenosis. Stroke, 22: 1485-90, 1991.
7. Murros K, Toole JF. Management of asymptomatic extracranial carotid artery disease. In: Welch KMA, Caplan LR, Reis DJ, Siesjö BK, Weir B. Primer on cerebrovascular diseases. New York, Academic Press, 1997: 762-5.

8. Wolf PA, Kannel WB, Sorlie P, et al. The Framingham study: Asymptomatic carotid bruit and the risk of stroke. JAMA, 245: 1442-5, 1981.

9. Mackey AE, Abrahamowicz M, Langlois $Y$, et al. For the Asymptomatic Cervical Bruit Study Group. Outcome of asymptomatic patients with carotid disease. Neurology, 48: 896-903, 1997

10. Executive Committee for the Asymptomatic Carotid Atherosclerosis Study. Endarterectomy for asympotomatic carotid artery stenosis. JAMA, 273: 1421-8, 1995.

11. Hill AB. Symposium: controversies in cerebrovascular disease 2. Should patients be screened for asymptomatic carotid artery stenosis? Canadian J Surg, 41: 208-13, 1998.

12. Hobson RW, Weiss DG, Fields WS, Goldstone J, Moore WS, Toene JB, Wright CB, and the Veterans Affairs Cooperative Study Group. Efficacy of carotid endarterectomy for asymptomatic carotid stenosis. N Engl J Med, 328: 221-7, 1993.

13. CASANOVA Study Group. Carotid surgery versus medical therapy in asymptomatic carotid stenosis. Stroke, 22: 122935, 1991

14. Mayo Asymptomatic Carotid Endarterectomy Study Group. Results of a randomized controlled trial of carotid endarterectomy for asymptomatic carotid stenosis. Mayo Clin Proc, 67: 513-8, 1992.

15. North American Symptomatic Carotid Endarterectomy Trial Collaborators. Beneficial effects of carotid endarterectomy in symptomatic patients with high-grade carotid stenosis. $\mathrm{N}$ Engl J Med, 325: 445-53, 1991.

16. Mayberg MR, Wilson SE, Yatsu F, Weiss DG, Messina L, Hershey LA, Colling C, Eskridge J, Deykin D, Winn HR. Carotid endarterectomy and prevention of cerebral ischemia in symptomatic carotid stenosis. Veterans Affairs Cooperative Studies Program 309 Trialists Group. JAMA, 266: 3289-94, 1991.

17. European Carotid Surgery Trialists' Collaborative Group. MRC European Carotid Surgery Trial: interim results for symptomatic patients with severe $(70 \%-99 \%)$ or with mild (029\%) carotid stenosis. Lancet, 337: 1235-43, 1991.

18. European Carotid Surgery Trialists' Collaborative Group. Endarterectomy for moderate symptomatic carotid stenosis: interim results from the MRC European Carotid Surgery Trial. Lancet, 347: 1591-3, 1995.

19. IMoore WS, Barnett HJM, Beeb HG, et al. Guidelines for Carotid Endarterectomy. A multidisciplinary consensus statement from the Ad Hoc Committee, American heart Association. Stroke, 26: 188-201, 1995.

20. Walker PM. Symposium: Controversies in cerebrovascular disease 3. Current indications for carotid endarterectomy. Canadian J Surg, 41: 214-7, 1998.

21. Baptista-Silva JCC. Endarterectomia da artéria carótida externa. Rev Bras Clin Terap, 24: 101-10, 1998.

22. Naylor AR, Bolia A, Abbot RJ, et al. Randomized study of carotid angioplasty and stenting versus carotid endarterectomy: a stopped trial. J Vasc Surg, 28: 326-34, 1998

23. Naylor AR, London NJM, Bell PRF. Carotid endarterectomy versus carotid angioplasty. Lancet, 349: 203-4, 1997.

24. Hobson II RW. Status of carotid angioplasty and stenting trials. J Vasc Surg, 27: 791, 1998

Endereço para correspondência:

Marcia Maiumi Fukujima Disciplina de Neurologia

Rua Botucatu, 740

CEP 04023-900 São Paulo (SP)

E-mail: maiumi@sti.com.br 